\title{
Kinetics and the Theoretical Aspects of Drug Release from PLA/HAp Thin Films
}

\author{
Innocent J. Macha ${ }^{1, a^{*}}$, Besim Ben-Nissan ${ }^{2, b}$ and Wolfgang Müller $r^{3, c}$ \\ ${ }^{1}$ Department of Mechanical and Industrial Engineering, University of Dar es Salaam, \\ P.O Box 35131, Dar es Salaam, Tanzania \\ ${ }^{2}$ School of Life Sciences, University of Technology Sydney, P.O. Box 123, Broadway, \\ NSW 2007, Australia
${ }^{3}$ Technische Universität Berlin, Fak. V, Institut für Mechanik, Lehrstuhl für Kontinuumsmechanik und Materialtheorie - LKM, Sekr. MS 2 Einsteinufer 5, D-10587 Berlin \\ aimacha@udsm.ac.tz, besim.ben-nissan@uts.edu.au, whmueller1000@gmail.com
}

\begin{abstract}
Keywords: kinetics, drug dissolution, thin films, coral-derived hydroxyapatite, antibiotic, drug delivery.
\end{abstract}

\begin{abstract}
The theory of dissolution kinetics of gentamicin from polylactic acid-hydroxyapatite thin film composites is spotlighted with the combination of diffusion and polymer degradation modeling. The use of various mathematical models, characterizing diffusion, dissolution or/and erosion prevalence as well as a mix of dissolution-diffusion rate processes were employed in order to compare theory with experimental data. A number of factors influence the release kinetics of gentamicin from medical drug release systems and devices. It is difficult to have a single mathematical model that takes all these factors into account. It is shown that the degradation of the polymer matrix plays the biggest role in the release kinetics of polymer-ceramics thin film composites. It was also observed that multistage drug release form these devices depends also on the degradation kinetics of the polymer matrix. The effect of $\mathrm{pH}$ and device sizes were not studied but could also be of interest in future studies.
\end{abstract}

\section{Introduction}

Slow drug release has been an important research subject in the field of drug delivery for decades. Drug release systems provide an outstanding alternative to conventional clinical therapies. The use of biodegradable materials such as polymer and calcium phosphates in designing drug release devices provides the outstanding capability of performing localized and controlled delivery of drugs to different parts of the host body. In order to allow for greater potency and less toxicity to healthy tissue, therapeutic agent release systems are required to control the release. Generally speaking in slow drug delivery devices, the mechanisms leading to drug release are linked to diffusion, dissolution and erosion of the matrix. However, additional interactions with the carrier can also modify the release kinetics. Furthermore, the physicochemical and morphological properties, the positioning of the drug within the porous network, its accessibility and its solubility are key parameters that govern the release kinetics and therefore the efficiency and efficacy of the treatment. There has been an enormous effort directed to the development of biodegradable materials that are capable of releasing drugs by reproducible and predictable kinetics $[1,2]$ to meet these demands.

Ceramics and other materials, such as polymers and biocomposites, have been proposed in the past, but it is difficult to shape them appropriately with adequate micro porosity in order to be fitted into any type and size of bone defect. Recently it has been demonstrated by Ben-Nissan and coworkers that marine shells with specific microspherical design offer desired functions for the delivery of Bisphosphonate (paminodrate) and antibiotic (Gentamicin) [3]. This has been possible by virtue of its unique structure and architecture of the foraminifera shells, which are extraordinarily difficult to manufacture with the current know-how [4]. 
Drug release from biodegradable polymer-ceramic composite is not only due to polymer degradation but also the diffusion of the drug entrapped into the polymer matrix, which plays an important role depending on the extension of the experiments. Langer [5] defined drug release from polymeric materials as the process in which drug solutes migrate from the initial position in the polymeric system to the polymer's outer surface and then to the release medium. There is much interest in being able to improve and predict precisely drug bioavailability and controlled drug release concentration to be within the therapeutic window in prolonged drug release [6]. The assessment of kinetic release from drug delivery devices provides the confidence for predicting the release behavior before the release systems are realized. This study focused on understanding theoretical phenomenon of drug dissolution based on the experimental results and critical assessment of the potential of theoretical modeling.

\section{Materials and Methods}

\section{Materials}

Coral skeleton samples were obtained from the Great Barrier Reef, QLD Australia by BenNissan and colleagues, Gentamicin sulfate, Clodronate (Dichloromethylenediphosphonic acid disodium salt), Chloroform diammonium hydrogen phosphate (NH4)2HPO4, 98\%), and sodium hypochlorite $(\mathrm{NaClO})$ were obtained from Sigma Aldrich, Castle Hill, Australia.

\section{Methods}

Drug loading to the PLA-films and PLA composites was performed based on our previous work [7, 8]. Drug release study from the devices were conducted under SINK conditions in phosphate buffered saline in a temperature controlled water bath shaker running at a constant speed of $100 \mathrm{rpm}$. Gentamicin concentrations in the solution were determined by using a Cary 100 UV-Vis spectrophotometer at the maximum absorbance of gentamicin-ophthaldialdehyde complex, $\lambda_{\max }=332 \mathrm{~nm}$, using procedures described in [7] .

\section{Theoretical}

On the basis of the pertinent literature mathematical models were conceived in order to predict the temporal development of the drug release over time. The pros and cons of these various models is discussed and a critical assessment of the potential of theoretical modeling is made.

It is worth mentioning that several factors influence the release mechanism, including the type of drug, environmental conditions during drug release as well as the geometry and dimensions of the drug delivery system, and preparation techniques, in order to mention just a few [9]. The assumption taken into consideration here is that the drug particles or particles loaded with drugs are dispersed randomly throughout the uniform polymer matrix with known geometry, such that the probability of finding drug at any point in the polymer matrix is constant at all positions within the matrix itself [10]. Based on previous studies, it was shown that the release behavior of a clinical active substance from degradable material follows the common pattern consisting of three stages $[11,12]$. However, a number of other studies have indicated that the behavior of drug release from biodegradable materials can follow two or four stages [9]. The common three stages of drug release could briefly be elucidated as follows:

Stage I: At time zero, water or buffer begins to hydrate the matrix, a process that happens quickly for the bulk eroding polymer matrices. As the matrix hydrates, encapsulated drug adjacent to the matrix surface or on the surface diffuses into the water or buffer in a phase reservoir in a phase typically known as "the initial burst." Its magnitude depends on the amount of drugs present adjacent to the matrix and on drug solubility.

Stage II: As the initial burst release takes place, degradation of the polymer chains begins, producing soluble oligomers and increasing chain mobility effectively leading to the formation of pores in the polymer matrix. It is believed that heterogeneous degradation of 
the polymer matrix starts with an amorphous region of polymer matrix leaving behind pores, creating a secondary surface porosity favourable for fluid transport phenomena and a further pathway for dissociation inside the composite. These pores appear to be essential for subsequent release of drugs [13]. The drug release by diffusion through the narrow or tortuous pores controls the mass transfer process. Graphical observation of the release profile indicates changes in the slope of the curve.

Stage III: Cumulative growth and coalescence of these pores as degradation of the polymer matrix progresses, as the results of water or PBS penetrates into the polymer network and hydrolyzes the polymer into more soluble oligomers, providing the channels for the drug to be able to diffuse towards the surface of a polymer matrix that would otherwise be too dense to allow their passage [13]. The drug is released progressively until complete polymer degradation, a process that depends on the polymer degradation behavior. In our case, the duration of the experiments for gentamicin loaded polymers were 15 [8], which is insufficient time to achieve complete full degradation.

For four stage drug release behavior it has been proposed to have a secondary boost before the final stages of drug release from the biodegradable matrix. These stages relate to the pure polymeric matrix and addition of the particulate matter as drug carrier introduces additional stages to the process, which will be discussed in the following section.

\section{Results and Discussion}

The release kinetic study was assessed by a model dependent method. Based on a number of different kinetic models available in literature, which described the overall release of drug from the dosage forms, the models were carefully selected and used to fit the release data [14-17]. Finally, data was fitted to seven different models: Zeroth order, first Order, Higuchi, Hixson-Crowell, Korsmeyer-Peppas and Reciprocal powered time. The correlation coefficients $\left(r^{2}\right)$ indicate that drug release kinetic of polylactic acid loaded with gentamacin (PLAGM) and Polylactic acid hydroxyapatite loaded with gentamicin (PLAHApGM) fitted with the power law model described by Korsmeyer-Peppas (Table 8). If this semi-empirical equation does not allow for the determination of all of the mechanisms involved in the release, it is still possible to determine the mechanisms of transport by considering two borderline cases, which correspond to distinct physical realities (when $n=0.5$ and $n=1)$. The coefficient $n$ obtained for PLAGM $(n<0.5)$ indicates that the release mechanism was mainly controlled by diffusion while the value obtained for PLAHApGM $(n>1)$ is characteristic of a number of mixed transport mechanisms including diffusion, possibly super case II kinetics as well as a release due to damage to the composite surface through dissolution. 
Table 1: Modelled dissolution characteristics of the mean dissolution profile [8]

\begin{tabular}{|llllc|}
\hline Model & Mathematical expression & & PLAGM & PLAHAPGM \\
\hline Zero order & $F=Q_{0}+k_{0} t$ & $r^{2}$ & 0.440 & 0.937 \\
& & $k_{0}$ & 0.125 & 0.070 \\
\hline \multirow{2}{*}{ First order } & $L n(1-F)=-k t$ & $r^{2}$ & 0.866 & 0.874 \\
\hline Higuchi & $F=a t^{1 / 2}+b$ & $r^{2}$ & 0.959 & 0.803 \\
\hline Hixson-Crowell & $1-(1-F)^{1 / 3}=k t$ & $a$ & 0.273 & 0.214 \\
\hline \multirow{2}{*}{ Korsmeyer-Peppas } & $F=k t^{n}$ & $k$ & 0.060 & 0.029 \\
\hline Baker Lonsdale & & $r^{2}$ & 0.992 & 0.962 \\
& $\frac{3}{2}\left[1-(1-F)^{2 / 3}\right] \times F=k t$ & $k$ & 0.282 & 1.315 \\
\hline \multirow{2}{*}{ Reciprocal powered time } & $\left(\frac{1}{F}-1\right)=\frac{m}{t^{b}}$ & $r^{2}$ & 0.945 & 0.727 \\
& & $b$ & 0.107 & 0.038 \\
\hline
\end{tabular}

$F=$ fraction of drug released up to time $t, r^{2}=$ square correlation coefficient, $Q_{o}, k_{o}, k, a, b, n, m$ and $b$ are parameters of the models.

\section{Stage I}

The initial very quick release (burst) of gentamicin (GM) can be assimilated as direct dissolution of drugs in water. It is shown in Figure 1 that the initial burst took only one week for gentamicin. At this stage it can be assumed that the release is purely governed by diffusion of drugs from polymer surfaces $[18,19]$.

\section{Stage II}

This step is driven by the internal diffusion of drugs impregnated within the matrix possibly in the porous part of the matrix generated during preparation. For gentamicin release from PLAHApGM samples, this stage is preceded by a "lag phase" which occurs between 1 and 2 hours of release. The presence of HAp loaded with gentamicin could in many ways hinders or slows down the release of gentamicin through these micropores. This stage is a bit slower release compared to the previous one due to the drug transporting through these small and narrow pores. 


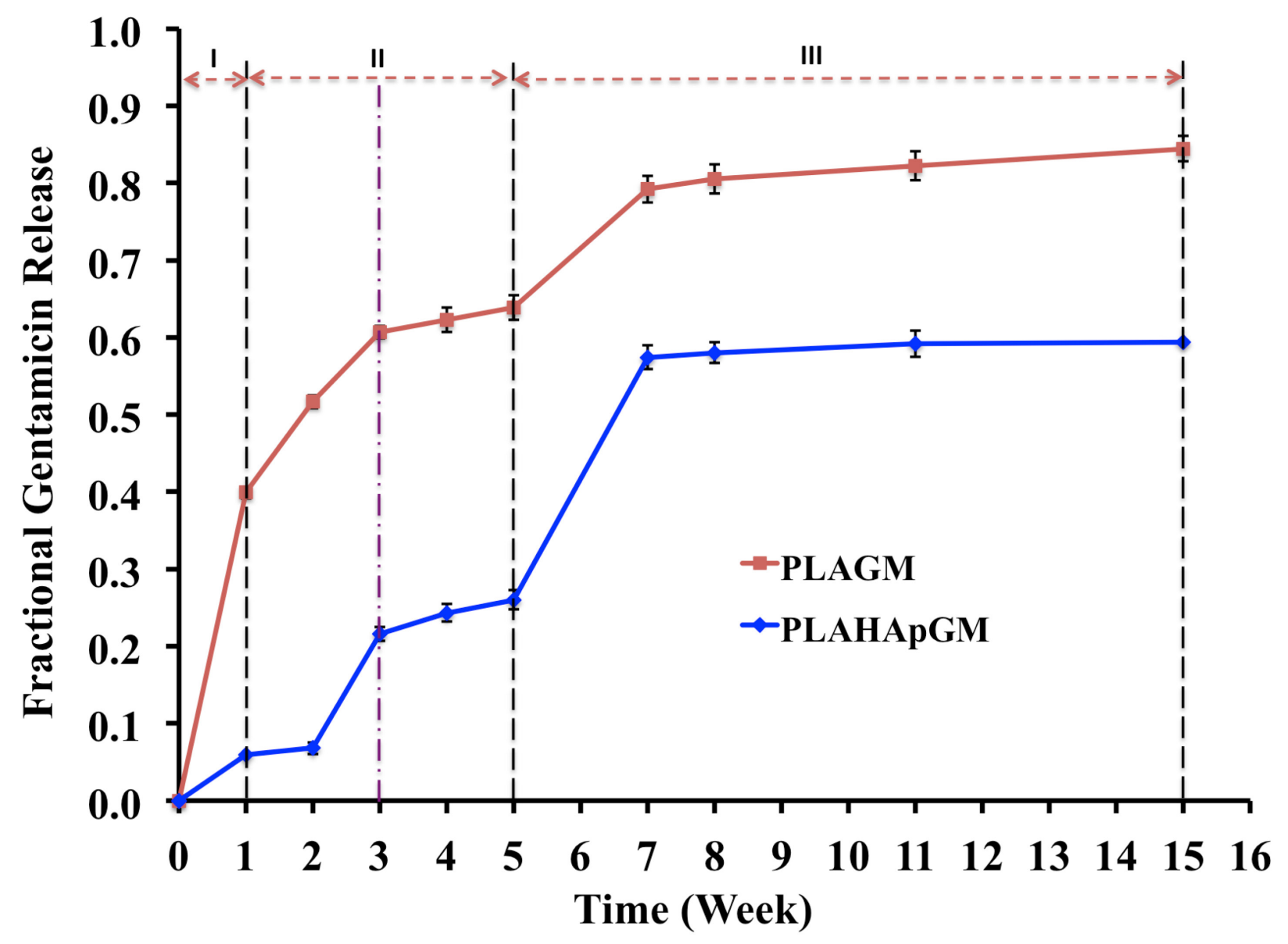

Figure 1: Fractional cumulative release of gentamicin from polylactic acid (PLA) thin film

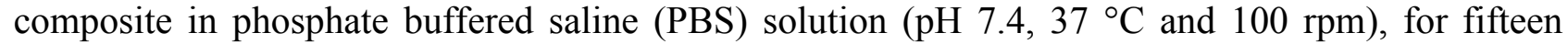
weeks. Error bars are mean standard deviation (SD) of triplicate experimental data

Table 2: Specific time frames for different release stages and their numerical values for gentamicin (Three stages)

\begin{tabular}{|c|l|c|c|}
\hline Type of drug released & \multicolumn{1}{|c|}{ Stages } & $\boldsymbol{t}_{\mathbf{0}}(\mathbf{w})$ & $\boldsymbol{t}_{\mathbf{f}}(\mathbf{w})$ \\
\hline \multirow{4}{*}{ Gentamicin } & Stage I (burst ext. release) & 0 & 1 \\
\cline { 2 - 4 } & Stage II (Internal release) & 1 & 3 \\
\cline { 2 - 4 } & Stage II (lag phase) & 3 & 5 \\
\cline { 2 - 4 } & Stage III (degradation) & 5 & $\infty$ \\
\hline
\end{tabular}

\section{Lag stage (Sub stage II)}

This is a 'lag stage or phase' for devices loaded with gentamicin. The lagging to release a significant amount of drug from the devices could be attributed to the $\mathrm{pH}$ change of entrapped, released acidic degradation products from the polymer matrix. The acidic environment would trigger unfolding of the encapsulated gentamicin until the degradation products dissolve and diffuse to the outer surface of the matrix providing a way for the drugs to be released [20].

\section{Stage III}

This is the terminal release stage for gentamicin-loaded devices. After a greater fraction of the drug is released, the remaining drug release is directly associated with polymer degradation [21]. There is the possibility of drug release by conventional diffusion at this stage, but it is considered to 
be negligible compared to that released by polymer degradation. In this study, it was observed that this stage was the slowest and steady stage drug release phase.

Dissolution profiles of gentamicin released from PLA film and PLA-Hydroxyapatite composite were compared by using a statistical difference factor (f1), and a similarity factor (f2). The test indicates that there is a significant statistical difference between the two kinetic profiles. This difference is confirmed by the release half-life $(t 50 \%)$ obtained for each material loaded with gentamicin. Thus, the release half-life from PLA film was obtained around 14 days while 124 days were necessary for PLA composite [8]. The difference between PLAGM and PLAHApGM regarding the amount of drug released is due to the fact that corolline HAp contains nano and meso pores in which drug goes into them and reduces drug accessibility during dissolution. HAp particles takes longer to dissociate than PLA.

\section{Conclusions}

Drug release mechanism from biodegreadable polymer-ceramic composites is a complex phenomenon which can not be defined by a single mathematical model. Precise prediction of release profiles is very important. Theoretical release mechanism presented in this paper took in consideration all aspects of polymer and ceramic behavior in phosphate baffered saline with an assumption of no strong affinity between drug and ceramics/polymer. The theory seemed to agree with the experimental data in our previous work. The research on PLA-ceramics thin film composites as the slow drug delivery devices reveals possible potential applications in biomedical field.

\section{References}

[1] Ginebra MP, Traykova T, Planell JA. Calcium phosphate cements as bone drug delivery systems: A review. Journal of Controlled Release. 113 (2006) 102-10.

[2] Habibe AF, Maeda LD, Souza RC, Barboza MJR, Daguano JKMF, Rogero SO, et al. Effect of bioglass additions on the sintering of Y-TZP bioceramics. Materials Science and Engineering: C. 29 (2009) 1959-64.

[3] Chou J, Valenzuela S, Green DW, Kohan L, Milthorpe B, Otsuka M, et al. Antibiotic delivery potential of nano- and micro-porous marine structure-derived beta-tricalcium phosphate spheres for medical applications. Nanomedicine. 9 (2014) 1131-9.

[4] Chou J, Ben-Nissan B, Green DW, Valenzuela SM, Kohan L. Targeting and Dissolution Characteristics of Bone Forming and Antibacterial Drugs by Harnessing the Structure of Microspherical Shells from Coral Beach Sand. Advanced Engineering Materials. 13 (2011) 93-9.

[5] Langer R. New methods of drug delivery. Science. 249 (1990) 1527-33.

[6] Santos A, Sinn Aw M, Bariana M, Kumeria T, Wang Y, Losic D. Drug-releasing implants: current progress, challenges and perspectives. Journal of Materials Chemistry B. 2 (2014) 6157-82.

[7] Macha IJ, Cazalbou S, Shimmon R, Ben-Nissan B, Milthorpe B. Development and dissolution studies of bisphosphonate (clodronate)-containing hydroxyapatite-polylactic acid biocomposites for slow drug delivery. Journal of tissue engineering and regenerative medicine. 11 (2017) 1723-31.

[8] Macha IJ, Cazalbou S, Ben-Nissan B, Harvey KL, Milthorpe B. Marine structure derived calcium phosphate-polymer biocomposites for local antibiotic delivery. Marine drugs. 13 (2015) 666-80.

[9] Siepmann J, Siepmann F. Mathematical modeling of drug delivery. International journal of pharmaceutics. 364 (2008) 328-43.

[10] Rothstein SN, Federspiel WJ, Little SR. A simple model framework for the prediction of controlled release from bulk eroding polymer matrices. Journal of Materials Chemistry. 18 (2008) 1873-80. 
[11] Rothstein SN, Federspiel WJ, Little SR. A unified mathematical model for the prediction of controlled release from surface and bulk eroding polymer matrices. Biomaterials. 30 (2009) 165764.

[12] Stephens D, Li L, Robinson D, Chen S, Chang H, Liu RM, et al. Investigation of the in vitro release of gentamicin from a polyanhydride matrix. Journal of controlled release : official journal of the Controlled Release Society. 63 (2000) 305-17.

[13] Fredenberg S, Reslow M, Axelsson A. Measurement of Protein Diffusion Through Poly(d,1Lactide-Co-Glycolide). Pharmaceutical Development and Technology. 10 (2005) 299-307.

[14] Mohammadi G, Barzegar-Jalali M, Valizadeh H, Nazemiyeh H, Barzegar-Jalali A, Siahi Shadbad MR, et al. Reciprocal powered time model for release kinetic analysis of ibuprofen solid dispersions in oleaster powder, microcrystalline cellulose and crospovidone. J Pharm Pharm Sci. 13 (2010) 152-61.

[15] Liechty WB, Kryscio DR, Slaughter BV, Peppas NA. Polymers for drug delivery systems. Annual review of chemical and biomolecular engineering. 1 (2010) 149-73.

[16] Costa P, Sousa Lobo JM. Modeling and comparison of dissolution profiles. Eur J Pharm Sci. 13 (2001) 123-33.

[17] Ford Versypt AN, Pack DW, Braatz RD. Mathematical modeling of drug delivery from autocatalytically degradable PLGA microspheres--a review. Journal of controlled release : official journal of the Controlled Release Society. 165 (2013) 29-37.

[18] Liechty WB, Kryscio DR, Slaughter BV, Peppas NA. Polymers for Drug Delivery Systems. Annual Review of Chemical and Biomolecular Engineering. 1 (2010) 149-73.

[19] Severino P, Santana MHA, Malmonge SM, Souto EB. Polymers for Drug Delivery Systems Formulations. Polimeros-Ciencia E Tecnologia. 21 (2011) 361-8.

[20] Zhu G, Mallery SR, Schwendeman SP. Stabilization of proteins encapsulated in injectable poly (lactide- co-glycolide). Nat Biotech. 18 (2000) 52-7.

[21] Cabezas LI, Gracia I, de Lucas A, Rodríguez JF. Novel Model for the Description of the Controlled Release of 5-Fluorouracil from PLGA and PLA Foamed Scaffolds Impregnated in Supercritical CO2. Industrial \& Engineering Chemistry Research. 53 (2014) 15374-82. 